\title{
LA INTEGRACIÓN DEL MOMENTO DE LA TÉCNICA EN EL PROCESO DE ESTUDIO DE CAMPOS DE PROBLEMAS DE MATEMÁTICAS
}

BOSCH, M. y GASCÓN, J.

Departament de Matemàtiques. Universitat Autònoma de Barcelona.

\section{SUMMARY}

This article provides a model of mathematical activity which describes it in terms of the study of fields of problems. This model helps us to analyze some didactical phenomena which are characteristic of the present systems of mathematics teaching. Special emphasis is devoted to the relation between the absence of so-called technique work, and some paradoxes about «mathematical creativity». A new didactical device is proposed, the workshop of mathematical practice, in order to bring the technique work alive in the classrooms as an integrating force in the study of mathematical problems. Finally, as an example, one of the workshops designed and tested is described.

El criterio de la creatividad no es solo la novedad; comprende también algo más -un nivel más elevado de acción, un mayor esfuerzo, una mayor eficacia.

Wladyslaw Tatarkiewicz, Historia de seis ideas (1976).

Todo trabajo de teorización o de ingeniería en diđáctica de las matemáticas presupone y utiliza necesariamente un modelo más o menos elaborado (aunque a menudo implícito) de la actividad matemática y, asimismo, una noción de lo que es «enseñar y aprender matemáticas». La explicitacion de estos modelos permite que sean cuestionados, contrastados empíricamente y reelaborados, por lo que debería constituir un punto de referencia en toda investigación de didáctica de las matemáticas.

Presentamos a continuación un trabajo de investigación realizado durante los cursos 90-91 y 91-92 (este último đentro del programa «Innovació en la docència universitària» del ICE de la UAB) que fue motivado inicialmente por el diseño y experimentación de una nueva actividad docente, las prácticas en matematicas. El conjunto de la investigación forma parte de un proyecto más amplio en dos sentidos: por una parte, visto como trabajo de ingeniería didáctica ${ }^{1}$, constituye el primer paso de un proceso que tiende hacia la instauración de los talleres de prácticas en el sistema de enseñanza de
Ias matemáticas; considerado por otra parte como contribución a la teoría de la didáctica de las matemáticas, pretende desarrollar y contrastar parcialmente algunos aspectos del modelo de los momentos didácticos. La construcción de este modelo, que sirve de fundamentación al proyecto, fue iniciada por Yves Chevallard en enero de $1991^{2}$.

Dividiremos la exposición en tres partes: en la primera enunciaremos de manera esquemática algunas de las tesis relacionadas con el modelo de la actividad matemática que tomamos como referencia; en la segunda utilizaremos los principales elementos teóricos presentados para analizar algunos aspectos de los actuales sistemas de enseñanza de las matemáticas; por último describiremos sucintamente uno de los talleres de prácticas que hemos diseñado y experimentado, a fin de ejemplificar el alcance «tecnológico» (por lo que se refiere a la ingenieráa didáctica) de nuestra investigación.

\section{ALGUNOS ASPECTOS DE NUESTRO MODELO DE REFERENCIA ${ }^{3}$}

\section{Análisis didáctico de la actividad matemática}

Postulamos que toda actividad matemática se puede analizar como actividad de estudio de campos de problemas, actividad que se lleva a cabo mediante la producción y utilización de lo que llamamos técnicas matemá- 
ticas o, más precisamente, técnicas de estudio de campos de problemas 4 .

En lo sucesivo, de acuerdo con este primer postulado, entenderemos que "hacer matemáticas» es producir y utilizar ciertas técnicas de estudio de ciertos campos de problemas. Con elio nos referimos a todos los «tipos» de actividades matemáticas en que se puede pensar, desde las más "teóricas» hasta las más "aplicadas», desde la del alumno de párvulos hasta la del investigador: todas podrán ser interpretadas como producciones o utilizaciones de diferentes técnicas matemáticas destinadas a estudiar diferentes campos de problemas (lo que puede incluir, en particular, su resolución).

Esta tesis inicial pone de manifiesto la importancia de la actividad de resolución de problemas dentro de nuestro modelo de la actividad matemática. Pero, dada la gran variedad de sentidos que tiene hoy día la expresión «resolución de problemas» dentro de la enseñanza de las matemáticas (Gascón 1992), debemos añadir algunas puntualizaciones.

a) La noción de problema debe entenderse aquí en un sentido muy general, que incluye desde los problemas escolares elementales, como la suma de dos fracciones o la resolución de una ecuación de segundo grado, hasta problemas de investigación que pueden no estar aún bien formulados por falta de una teoría adecuada, sin excluir los problemas considerados «teóricos», como por ejem. plo enunciar y demostrar un teorema o axiomatizar una teoría.

b) No se conciben los problemas aislados, sino sólo como integrantes de campos de problemas. Los matemáticos no trabajan generalmente para resolver problemas desconectados entre sí; siempre postulan que el problema que están estudiando es un representante de cierto campo de problemas, aunque no conozcan de dicho campo más que algunos problemas emblemáticos. Su trabajo consiste entonces en producir los medios mate. máticos (las técnicas) necesarios para llevar a cabo el estudio de este campo, y no sólo la resolución del problema particular considerado.

c) Lo que es especifico de la actividad matemática no es la mera resolución de problemas sino, como ya hemos dicho, la producción de técnicas de estudio de campos de problemas. Las técnicas a las que nos referimos son "técnicas» en el sentido general de "maneras de hacer» algo, abarcando desde las más algorítmicas y visibles (como el algoritmo de resolución de las ecuaciones de segundo grado) hasta las menos conscientes o explicitables (como las que se utilizan para modelizar matemáticamente cierto sistema dado o para demostrar cierta proposicion $)^{5}$.

d) El estudio de campos de problemas, esto es, la utilización y producción de técnicas matemáticas, requiere la existencia de desarrollos teóricos y tecnologicos (en el sentido de discursos -logos- sobre las técnicas) cuya principal funcion es la de explicar, justificar y relacionar entre sí las técnicas manipuladas y los campos de problemas abordados, para asegurar tanto el control de los resultados de la actividad como su presentación y legibilidad. Debemos recaicar que estos elementos justificativos y explicativos no son «teóricos» por naturaleza, sino por la función que asumen (justificativa, explicati$\mathrm{va}$, etc.) respecto al estudio de los campos de problemas que se considera como "núcleo" de la actividad. Así un teorema puede ser considerado como una parte de un problema (en una actividad en la que nos proponemos demostrarlo, por ejemplo) y puede también ser considerado como elemento teórico, si asume el papel de legitimador y justificador de cierto tipo de técnicas que se utilizan para estudiar otros problemas.

e) Existe una dependencia mutua entre los diversos productos u objetos de la actividad matemática: de la misma manera que un desarrollo térico puede generar nuevos campos de problemas y nuevas técnicas de estudio, también el desartollo interno de las técnicas origina la ampliación de los campos de problemas e incide sobre la teoría asociada, puesto que ésta debe ser capaz de justificar, describir e interpretar las técnicas matemáticas desarrolladas y los campos de problemas ampliados.

En este modelo de la actividad matemática no cabe, por tanto, considerar una teoría matemática, ni un campo de problemas, ni siquiera un simple problemo o una técnica matemática de manera aislada e independiente del resto de elementos que configuran la actividad. Hablar de un campo de problemas presupone tener en cuenta al mismo tiempo-aunque sólo sea potencialmente- una o más técnicas de estudio del campo (sin la existencia de las cuales éste no tendría sentido), así como presuponer un entorno teórico donde se definen, justifican e interrelacionan los objetos matemáticos que componen técnicas y problemas. Recíprocamente, cuando hablamos de una teoría matemática, estamos considerando implícitamente los campos de problemas (y las técnicas de estudio) que han cristalizado o que se sintetizan en la teoria considerada, así como aquellos campos de problemas ( $y$ aquellas técnicas) que la teoría permite enunciar y fundamentar.

Hemos presentado así un esbozo de un modelo epistemologico general de la actividad matemática que enfatiza la interrelación entre los tres elementos siguientes: el desarrollo de las técnicas matemáticas; la evolución que sufren los campos de problemas al ser estudiados con dichas técnicas; y la construcción de las teorías matemáticas asociadas, que permiten describir, interpretar y justificar los distintos elementos que componen técnicas y campos de problemas. Se trata, en síntesis, de un modelo compatible con muchas de la investigaciones contemporáneas en historia y epistemología de la ciencia $^{6}$ que, aunque defienden modelos distintos de los mecanismos del desarrollo del conocimiento científico, se oponen frontalmente al modelo ingenuo y espontáneo que puede ser descrito como "lineal y acumulativo» (Gascón 1993).

\section{Análisis didáctico dela noción de kenseñar matemáticas»}

Uno de los ragos esenciales de la điđáctica fundamental, iniciada por Guy Brousseau en los años setenta, consiste 
en retrotraer el objeto "primario» de estudio de la didáctica de las matemáticas a la actividad matemática en sí misma y a los modelos implícitos de dicha actividad (y de sus objetos) que se construyen inevitablemente durante el proceso de enseñanza. De esta forma, las características del sujeto que aprende matemáticas y del que las enseña, así como la forma de aprenderlas y enseñarlas, pasan a ser objetos de estudio «secundarios» (no por ello menos importantes) porque su estudio supone un anălisis previo de la actividad matemática que los define como enseñante y estudiante. En particular, eI significado de «enseñar matemáticas» $\mathrm{y}$ «aprender matemáticas» se vuelve inseparable del modelo epistemológico de la actividad matemática que se asuma o elabore, por lo que tales expresiones dejan de tener el carácter de «noción primitiva» que tenían en la didáctica clásica, en la que su significado sólo dependía de determinados modelos psicológicos o pedagógicos del aprendizaje, independientes de los conocimientos matemáticos invoIucrados e independientes, por lo tanto, de los fenómenos didácticos correspondientes.

Situánđonos explícitamente dentro de la didáctica fundamental y apelando al modelo de la actividad matemática que hemos tomado como referencia, consideramos que la enseñanza de las matemáticas debe analizarse en términos de estudio de campos de problemas, técnicas matemáticas y teorías matematicas asociadas. En otras palabras, postulamos que enseñar matemáticas (o cierto ámbito de la actividad matemática) es posibilitar que los alumnos sean capaces de estudiar de manera autónoma un conjunto seleccionado de campos de problemas, esto es, que lleguen a poner en práctica y desarrollar ciertas técnicas de estudio de dichos campos.

Partiremos asi de la suposición de que todo proyecto de enseñanza de las matemáticas se establece (aunque no aparezca bajo esta forma) alrededor de un corpus de campos de problemas, con un conjunto de técnicas de estudio asociadas inicialmente a cada campo y, en cada caso, el entorno teórico necesario para desarrollar la actividad. Dicho proyecto se determina siempre a partir de los distintos elementos que configuran la actividad matemática que se quiere enseñar. En consecuencia, sea cual sea el sistema didáctico observado, y sea cual sea la forma en que el propio sistema describe y presenta lo que en él se ensefia (o se quiere enseñar), siempre podremos reformularlo en los términos anteriormente descritos.

Es importante insistir en que el modelo general de la actividad matemática que asumimos no determina unívocamente el sentido de la noción «enseñar matemáticas» que hemos adoptado; pero ello no significa que no determine absolutamente nada. Al contrario, cuando lo utilizamos para describir, analizar y explicar el sistema de enseñanza de las matemáticas, el modelo determina nada menos que el tipo de preguntas que nos podemos formular y la forma que pueden tomar las respuestas (aunque no el contenido de éstas). En nuestro caso tendrá sentido plantearse preguntas tales como: ¿Cuáles son los campos de problemas que se enseñan en tal sistema? ¿Qué técnicas se utilizan para la exploración de estos campos? ¿Qué teoría servirá a la clase para justificar la aplicación y alcance de estas técricas? ¿Cómo (con qué clase de problemas) se puede enseñar una técnica determinada? ¿Hasta qué punto es necesario que el estadiante la domine? ¿Con qué otras técnicas la relacionará el profesor? ¿Cómo se «evalúa el građo de dominio de una técnica? ¿Qué variaciones de la misma deberá utilizar el estudiante? ¿Qué estatuto se otorga a un determinado objeto matemático: se enseña como parte de una técnica, de una justificación teórica, como componente de un campo de problemas? ¿Qué aspectos del estudio de un campo de problemas se dejan para la gestión personal del alumno? ¿Qué otras son únicamente asunto deI profesor? ¿Qué aspecto de la actividad matemática ha sido capaz de realizar el alumno cuando decimos de él que ha entendido o adquirido un concepto determinado? Etcétera.

Las respuestas dadas a estas y otras preguntas es lo que nos permite caracterizar los diferentes «contenidos matemáticos» que se enseñan, sus interrelaciones y el estatuto que éstos adquieren en las actividades matemá ticas presentes en cuałquier sistema de enseñanza. Entre otras cosas, los tipos de preguntas presentados permiten delimitar en cada caso distintos modelos específicos relativos a cada ámbito (o tema) concreto de la matemática que se enseña o se quiere enseñar. Así, por ejemplo, si nos queremos referir (dentro de la teorización) a la «enseñanzá o aprendizaje de la proporcionaljdad» en cierto nivel educativo, deberemos especificar en particular qué tipos de problemas aparecen como representantes del campo de problemas de proporcionalidad en el sistema de enseñanza considerado; bajo qué forma se presentan; qué técnicas están en la base de lo que se entiende por «resolver problemas de proporcionalidad» o, simplemente, «utilizar la proporcionalidad»; con qué nociones y desarrollos teóricos se interpretan y justifican; etc. Queremos insistir en que los modelos específicos a los que nos referimos, aunque coherentes con el modelo general adoptado y sólo formulables dentro de él, no están completamente determinados por éste: son fruto de una elección sujeta a muchas restricciones pero que conserva algunos grados de libertad. Un ejemplo de lo que llamamos modelo especifico es el que elaboró Brousseau para analizar los fenómenos didácticos relativos a la enseñanza de los números decimales (1981, pp. 40 y ss.) y está en la base de lo que se denomina situación ("a-didáctica») fundamental relativa a un saber espe. cúfico.

\section{LOS SISTEMAS ACTUALES DE ENSEÑANZA DE LAS MATEMATICAS}

\section{Diferentes formas de entender la expresión de «enseñar matemáticas»}

En los sistemas actuales de enseñanza de las matemáticas a nivel universitario, el principal criterio explícito utilizado para describir lo que se quiere enseñar es un criterio relativo a las teorías matemáticas o a distintos elementos de las mismas. El proyecto de enseñanza se establece entonces a partir de la explicitación inicial de los elementos teóricos que el profesor debe enseñar y el estudiante aprender, quedando en gran parte implícitos 
los campos de problemas que se explorarán durante el curso y el tipo de desarrollo de las diversas técnicas matemáticas necesarias para realizar dicha exploración. Notemos sin embargo que estos campos de problemas y estas técnicas no dejan de estar presentes en el proceso de enseñanza: son los problemas que el alumno tiene que aprender a resolver y que se utilizarán, en gran parte, para la evaluación del curso. Lo que decimos es que no están presentes explícitamente como objetivo đel curso ni en la estructuración del mismo. Esta situación es característica de lo que hemos denominado paradigma teoricista (Gascón 1992).

La actividad de estudio de campos de problemas, en cuanto objetivo explícito del proceso de enseñanza, tampoco aparece en el llamado paradigma modernista (que tiene mayor implantación en los niveles de enseñanza no universitaria), el cual se caracteriza por la identificación que realiza entre «actividad matemática» $\mathrm{y}$ «exploración de problemas». La principal estrategia de enseñanza consiste entonces en enfrentar al estudiante con diferentes problemas aislados y descontextualizados, dejando implícitos (e incluso «reprimidos») tanto los campos de problemas como las técnicas matemáticas pertinentes para su estudio.

Podemos observar en general que, aunque por razones distintas, se evita en ambos casos explicitar suficientemente la materialidad de la actividad matemática que el estudiante debe llegar a realizar por sí mismo al final del proceso de enseñanza. Señalaremos brevemente algunas de las consecuencias más importantes de esta tendencia. A nivel universitario, donde se tiende a identificar «enseñar matemáticas» con «enseñar teorías acabadas», se da preponderancia a una formación "generalista» en detrimento de una formación más profundizada y especializada. Mientras que en los niveles no universitarios, en la medida en que se identifica "enseñar matemáticas» con enseñar la actividad de explorar «libremente» (libre también de las teorías y de las técnicas matemáticas) problemas aisiados, se descuida la relación entre la resolución de problemas y los elementos teóricos asociados. En ambos paradigmas se ignoran las funciones epistemológicas y didácticas del desarrollo de las técnicas matemáticas en el estudio de campos de problemas (Gascón 1992).

\section{Estudio «profundizado» de un campo de problemas matemáticos}

Utilizando el modelo de la activiđad matemática presentado anteriormente, es posible ahora tomar en consideración las funciones del desarrollo potencial de las técnicas matemáticas en el proceso de estudio de un campo de problemas. En este sentido, empezaremos por distinguir entre lo que llamaremos estudio profundizado de un campo de problemas y el estudio exploratorio de problemas inicialmente aislados. Por una parte, y como ya indica el adjetivo «exploratorio», se trata del estudio que se realiza al abordar o entrar en contacto con un campo de problemas del cual sólo se conocen algunos representantes. Es el tipo de actividad que realizamos cuando nos enfrentamos por vez primera a un problema con unas cuantas técnicas preestablecidas, o cuando utilizamos alguna técnica matemática con la que estamos poco familiarizados. Por su parte, el estudio profundizado, que supone necesariamente un estudio exploratorio previo, va más allá de la aplicación puntual de técnicas dadas a problemas prefijados y va más allá incluso đe la mera resolución de los problemas abordados: se fundamenta en el dominio y desarrollo de las técnicas matemáticas manipuladas, con la posibilidad de dar origen a nuevas técnicas (debido a mođificaciones de las técnicas conocidas) y de que aparezcan nuevos problemas dentro del campo inicial cuyo estuđio requerirá a su vez ciertas modificaciones técnicas. Es en este «nivel» de estudio donde aparece la necesidad de elementos teóricos «nuevos» (aunque sólo sean nuevos para los actores de la actividad) destinados a interpretar y justificar las nuevas técnicas y sus interrelaciones. Si entendiésemos el proceso de estudio de campos de problemas únicamente como un estudio exploratorio (cuyo objetivo radicaría en la mera resolución de los problemas abordados), nos sería difícil dar cuenta del porqué la actividad matemática es capaz de evolucionar, plantearse nuevos objetivos y desarrollar sus instrumentos. Al interpretar la actividad matemática como actividad de estudio đe campos de problemas matemáticos, debemos prolongar la exploración de problemas (y su resolución) con el trabajo de desarrollo de las técricas que es productor de técnicas nuevas, «descubridor» de nuevos campos de problemas y elaborador de las nociones teóricas que justifican, sintetizan e interrelacionan los distintos objetos de la actividad.

Postulamos que, mientras el estudio exploratorio de problemas cumple un objetivo điđáctico funđamental de toma de contacto y familiarización con las teorías, Ias técnicas matemáticas y los campos de problemas que se quieren enseñar, un estudio profundizado de ciertos campos de problemas constituye un complemento imprescindible en toda formación matemática.

Esta tesis coincide aproximadamente con la especificación en el caso de las matemáticas de una idea general expresada por Whitehead en The Aims of Education (1929), según la cual la formación en cualquier disciplina científica debería contemplar, al lado de una visión amplia, general y forzosamente superficial del saber que se enseña, una introducción a la propia actividad científica en cuestión mediante el estudio especializado y profundo de un parcela reducida de esta ciencia. Esta afirmación se opone a la idea dominante en nuestra cultura escolar según la cual los estudios «profundizados y especializados» son propios de los niveles superiores de formación (por ejemplo, el tercer ciclo universitario) y no deberían integrarse en la formación universitaria básica, y mucho menos en los niveles preuniversitarios? Mientras que la tesis de Whitehead implica únicamente que ambos aspectos de la formación matemática han de estar unidos en cierta proporción, la nuestra, al especificar la noción de «estudio profundizado de un campo de problemas» y contraponerla al mero «estudio exploratorio», nos permitirá además explicar el porqué y ei cómo de esta complementariedad. En lo quie sigue de esta sección expondremos el porqué, dejando el cómo para más adelante. 


\section{Los momentos del proceso didáctico}

La necesidad de distinguir diferentes dimensiones en la actividad matemática, esto es, en el trabajo llevado a cabo durante el estudio de campos de problemas, nos lleva a aumentar la complejidad del modelo presentado en la sección anterior. Partiendo de la descripción de la actividad matemática como utilización y producción de técnicas de estudio de campos de problemas, pasamos a estructurar y caracterizar este proceso de estudio en, al menos, tres momentos diferentes, que describimos brevemente a continuacion. Usamos la palabra «momento» no en el sentido temporal y cronologico, sino en el sentido de "aspecto" o "dimensión" del proceso de estudio de un campo de problemas. Esto significa que no debemos considerar los tres momentos en que se estructura dicho proceso ni como tres formas diferentes de estudiar campos de problemas ni como tres etapas sucesivas en el estudio de cada campo. Se trata de tres "dimensiones ideales» que se integran de manera compleja en toda actividad matemática concreta, esto es, en todo proceso de estudio de un campo de problemas.

Ya hemos dicho que el estudio exploratorio de problemas aislados cumple un importante objetivo didáctico: constituye esa etapa o dimensión del proceso didáctico en la que el estudiante se ve enfrentado a problemas totalmente nuevos para él, y en la que debe manipular por primera vez las técnicas pertinentes para su estudio. Llamaremos momento exploratorio a esa dimensión de la actividad matemática que aparece, reiteradamente, siempre que el actor de la actividad (sea estudiante primerizo o matemático profesional) aborda un problema de un nuevo campo o cada vez que un desarrollo técnico le lleva a plantearse un problema que requiere utilizar una técnica diferente de la habitual.

Ahora bien, también hemos visto que para ir más allá de la exploración del campo de problemas abordado es necesario partir de un dominio de las técnicas suficientemente robusto para permitir su desarrollo y variacion. Llamaremos trabajo de la técnica al trabajo subyacente de manipulación de las técnicas, y momento de la técnica a la dimensión del proceso de estudio en la que se sitúa este trabajo. Por último, denominamos momento té́rico a cada una de las etapas del estudio en las que predomina el trabajo de presentación, descripción, justificación e interpretación de los distintos elementos que configuran las técnicas matemáticas y su entorno; en otras palabras, al trabajo teórico asociado al estudio de un campo de problemas (Chevallard 1992).

Cualquier análisis más detallado de la actividad matemática (tenemos un ejemplo en el realizado por James Ritter, en Serres 1989) mostraría que la articulación funcional de estos tres momentos requiere que el momento de la técnica juegue el papel de momento integrador del proceso de estudio de campos de problemas, esto es, que aparezca como desarrollo natural del momento exploratorio y como fuente de nuevas necesidades teóricas. Esta función integradora es la primera razon, y también la más básica, de por qué el estudio profundizado de algunos campos de problemas debe complementar el estudio exploratorio.
Gracias a la estructuración del proceso de estudio de campos de problemas en tres momentos diferentes, disponemos ahora de un instrumento teórico suficiente para interpretar algunos fenómenos didácticos muy visibles en los actuales sistemas de enseñanza de las matemáticas y que se mantenían inexplicados hasta la fecha. Estas interpretaciones aportarán razones complementarias para apoyar el postulado de la necesidad del estudio profundizado de ciertos campos de problemas como complemento de toda formación matemática.

\section{Trabajo de la técnica y "creatividad matemática»}

Partimos de la hipótesis de que el estudio profundizado de un campo de problemas sólo se puede llevar a cabo a partir del dominio de algunas técnicas matemáticas pertinentes. No es suficiente, en efecto, la utilización correcta, ni siquiera reiterada de estas técnicas; se necesita además dominarlas hasta el punto de poderlas modificar para adaptarlas a nuevas situaciones (es decir para proseguir con el estudio de nuevos problemas). Quien realmente domina las técnicas, el experto, actúa de tal modo que no es consciente de que las está usando efectivamente, y esto le permite no sólo usarlas de manera flexible, sino además incluirlas en actividades más amplias y complejas 8 .

Lo anterior puede interpretarse diciendo que sólo el experto es capaz de una actividad matemática creativa, definiendo ésta como aquella actividad que permite, en primer lugar, producir técnicas relativamente nuevas a partir de variaciones de las ya conocidas y, en segundo lugar, sintetizar los diferentes aspectos del conocimiento matemático desarrollado para configurar cierta visión global del mismo. Un estudio únicamente exploratorio de los diferentes aspectos del universo matemático (que comporta forzosamente una utilización rígida de las técnicas) no sólo incapacita al estudiante para dirigir las técnicas manipuladas hacia el estudio de nuevos problemas, también le impide relacionarlas entre sí e interpretarlas como parte de un todo más amplio. Porque es sólo en caliđad de experto -aunque sólo sea de una pequeña parcela de las matemáticas- como podrá el estudiante «apreciar la exacta formulación de las ideas generales» (Whitehead 1929, p. 30).

En síntesis, podemos concluir que el trabajo de la técnica es condición imprescindible para llevar a cabo una actividad matemática creativa, tanto en el sentido de "creadora» de nuevas técnicas como en el de «generadora» de nuevas relaciones entre técnicas, teorías y campos de problemas, con la formulación consiguiente de «ideas generales» al respecto.

\section{Ausencia institucional del trabajo đe la técnica}

Si nos referimos a las actividades docentes «públicas», por oposición a las privadas que realiza el alumno individualmente fuera del aula, postulamos que en los actuales sistemas de enseñanza de las matemáticas es difícil que la actividad de estudio de campos de problemas se lleve de forma sistemática más allá del nivel 
exploratorio. No hay ningún dispositivo didáctico institucionalizado que fomente el trabajo técnico y permita al estudiante pasar de la simple exploración al estudio profundizado de ciertos campos de problemas.

Esto no significa que el sistema de enseñanza de las matemáticas ignore absolutamente el trabajo de la téc. nica. Lo que queremos decir es un poco más complejo. Por una parte, en los niveles superiores de la enseñanza (a nivel de diplomatura o licenciatura), el trabajo de la técnica no tiene suficiente visibilidad y oficialidad, a diferencia de los momentos exploratorios y a diferencia, sobre todo, de los diferentes momentos teóricos. Por otra parte, en los niveles preuniversitarios, el trabajo de la técnica es visto como una amenaza para la exploración «libre» y «creativa» de problemas. Y finalmente, allí donde se ha impuesto el grito defensivo de «jvolver a lo básico!» (especialmente a nivel de enseñanza primaria), se tiende a enfatizar únicamente los aspectos más rudimentarios del momento de la técnica por ser éstos los más algoritmizables y fácilmente evaluables (Gascón 1992). En ningún caso se ha llegado a materializar, mediante un dispositivo didáctico oficial, la conexión entre los diversos momentos del estudio de un campo de problemas; más bien se contrapone el dominio robusto de las técnicas a la actividad matemática creativa, como si fuesen dos aspectos de la actividad matemática incompatibles entre sí. Llamaremos ausencia institucional del trabajo de la técnica a este fenómeno.

Esta ausencia proviene, en principio, del hecho de que la cultura tiende a considerar peyorativamente el trabajo de la técnica, trabajo considerado como "meramente mecánico y repetitivo». Pero una observación más atenta debería hacer patente que esta «tepresión» de todo lo que pueda parecer rutinario en la enseñanza de las matemáticas (represión que se acentúa en los niveles educativos superiores) proviene en realidad de la necesidad del sistema de enseñanza de dar ta impresión (tanto al observador exterior como a sus propios actores) de que el trabajo matemático que se realiza es un trabajo «creativo», en el sentido ingenuo de «no rutinario». En nuestra cultura se confunde «actividad creativa» con "actividad no rutinaria», sienđo quizá el carácter «libre» y «espontáneo» de ésta el que le confiere una cierta apariencia de creatividad.

Esta necesidad de creatividad aparente deja al alumno en una situación de incertidumbre permanente, en la que se ve enfrentado constantemente con problemas nuevos que le exigen la utilización de técnicas también nuevas. En tal situación, el alumno sólo puede alcanzar un dominio muy débil de las técnicas que se le presentan, lo que le incapacita, paradójicamente, para una actividad matemática genuinamente «creativa» en el sentido que hemos explicado en la sección anterior ${ }^{9}$.

La ausencia institucional del trabajo de la técnica origina, por tanto, importantes contradicciones y disfunciones dentro del sistema de enseñanza: por una parte, se otorga gran valor a la «creatividad» en la actividad matemática pero, por otra parte, no se poseen los medios necesarios para hacerla existir en el aula. Estas disfunciones suelen traducirse en una frustracion general: en unos casos se acaba abandonando explícitamente un objetivo que la fuerza de los hechos presenta cada vez como más inasequible, cambiándolo por otro mucho menos «prestigioso" que consiste en hacer que el alumno domine ciertas técnicas aisladas, sin pretender que sepa desarrollarlas ni ser capaz de delimitar su ámbito de aplicación; en otros casos, aunque se mantenga explícitamente el objetivo inicial de enseñar una actividad matemática «creativa», se observa un repliegue implícito hacia posiciones menos ambiciosas y más acordes con el tipo de actividad matemática efectivamente presente en el aula (fenómeno que se pone especialmente de manifiesto, a la larga, en el proceso de evaluación).

\section{EL TALLER DE PRÁCTICAS MATEMÁ- TICAS}

Basándonos en los análisis anteriores, hemos diseñado un nuevo dispositivo didáctico, el taller de prácticas matemáticas, donde el estudiante pueda aprender a hacer, de manera oficial, el tipo de trabajo matemático necesario para profundizar en el estudio de algunos campos de problemas concretos. La función principal del taller de prácticas es hacer vivir el momento de la técnica dentro del horario lectivo, con el mismo rango que los demás momentos del proceso didáctico que ya tienen dispositivos institucionalizados propios.

Debería parecer lógico que un sistema de enseñanza que valora extraordinariamente la "creatividad» de la actividad matemática (en los dos sentidos indicados) oficialice la enseñanza del trabajo de la técnica como condición imprescindible para el desarrollo creativo de dicha actividad. Pero debemos tener en cuenta los efectos, en la cultura escolar, de la confusión entre «actividad creativa» $\mathrm{y}$ "actividad no rutinaria». Lo que proponemos como actividad inicial de los alumnos en el taller de prácticas es aparentemente un tipo de trabajo matemático muy insignificante y humilde, que carece hoy día de suficiente legitimidad cultural para vivir con normalidad en las aulas. Nos tenemos que apoyar pues en su pertinencia epistemológica y didactica, es decir, en su necesidad para el desarrollo de la actividad matemática en general y del proceso didáctico en particular, si queremos darle cabida en el mundo oficial de la enseñan$\mathrm{za}$, como paso previo para que viva de manera natural en manos del estudiante (primero en las instituciones docentes y luego fuera de ellas).

La organización del taller de prácticas parte de la elección de un campo de problemas determinado dentro de los distintos campos que configuran el plan de estudios. Su objetivo es que los estudiantes puedan adquirir, para llevar a cabo el estudio del campo de problemas elegido, un dominio suficiente de ciertas técnicas matemáticas de forma que puedan desarrollarlas y variarlas espontáneamente a medida que se enfrentan con nuevos problemas del campo. Cada taller se estructura en tres o cuatro sesiones; cada sesion tiene una duración de cuatro horas y posee un subobjetivo propio. La áltima media hora de cađa sesión está destinada a la realización de una microprueba individual en la que el estudiante debe resolver 
de manera ágil y fiable, y en un tiempo relativamente limitado, un problema similar a los estudiados durante la sesión $^{10}$.

Al principio de cada sesión se distribuye a los estudiantes el «material de prácticas» del tafler. Es este material el que debe dirigir la marcha del trabajo, aunque se podrá ver complementado ocasionalmente con algunas intervenciones del profesor. Dicho material presenta, en cada sesión, algunos problemas del campo escogido que se pueden abordar con una misma técnica (podemos decir que representan un "subcampo» del campo considerado). Los estudiantes deben resolver inicialmente estos problemas mediante la utilización de una técnica conocida y progresar paulatinamente con técnicas originadas por variación de la técnica inicial. El principal objetivo del estudio no debe ser únicamente la resolución de cada problema, ni tampoco el mero dominio de las técnicas utilizadas; se pretende que los estudiantes consigan desarrollarlas y modificarlas a lo largo del estudio, hasta convertirse en «pequeños expertos» en la actividad.

En el diseño de un taller de prácticas tiene una importancia capital la elaboración del «material de prácticas». En primer lugar, en su elaboración se realiza el trabajo de explicitación y descripción del campo de problemas que se estudiará en clase, de las técnicas que han de permitir al estudiante llevar a cabo el estudio, de los desarrollos y variaciones técnicas que este estudio requiere, y del entorno teórico necesario. De aquí surge en realidad la explicitación del modelo específico đel ámbito matemático que se pretende generar (modelo al que nos referíamos con anterioridad). En segundo lugar, es este material el que debe hacer aparecer, en manos de los estudiantes, la posibilidad e incluso la necesidad de modificar y generalizar las técnicas utilizadas para poder seguir resolviendo los problemas que se les presentan.

En el lenguaje de la teoría de las situaciones didácticas, podría decirse que el «material de prácticas» debe constituir el núcleo de una situación «a didáctica» específica de la técnica matemática que los estudiantes deben desarrollar. Esta formulación no debe esconder que el elemento central del taller no son las técricas estructuradoras, sino la actividad matemática más compleja que el desarrollo de estas técnicas posibilita. Los talleres de prácticas se deben concebir siempre como un dispositivo didáctico más, en el que se desarrolla un aspecto o momento de la actividad matemática estrechamente dependiente de los demás. Olvidar esta función, a la vez subordinada y articuladora, nos podría llevar a una excesiva «didactificación» de las técnicas enseñađas, lo que significaría a la larga identificar la función de los talleres de prácticas con la enseñanza de la utilización de técnicas matemáticas.

\section{Breve descripción de un taller de prácticas experimentado}

Presentamos aquí, a título de ejemplo, uno de los talleres de prácticas diseñados y experimentados durante el curso 91-92 con estudiantes de primer curso de Análisis de la licenciatura de matemáticas de la UAB"l. La expe- rimentación ha sido llevada a cabo por un equipo formado por los tres profesores de la asignatura (un profesor de «teoría» y dos de (problemas») más los autores. Nosotros hemos sido los responsables de la coordinación y los encargados del diseño, el seguimiento y la evaluación de todo el proceso.

Este taller ha sido diseñado con el proposito de que los estudiantes lleven a cabo el estudio profundizado de un pequeño campo de problemas situado en el (inmenso) campo de las ecuaciones diferenciales lineales. El primer objetivo del taller es que el estudiante llegue a dominar de una manera robusta las distintas técnicas que aparecen, a fin de que sea capaz de realizar por sí mismo pequeñas variaciones de dichas técnicas. El segundo objetivo consiste en posibilitar, a partir de las variaciones relevantes de las técnicas, que surjan en manos de los estudiantes las consiguientes ampliaciones del campo de problemas y de las necesidades teóricas asociadas. El «material de prácticas» elaborado para este taller lleva por título Sèries de potències $i$ equacions diferencials (Bosch 1992).

La duración total del taller fue de tres sesiones de cuatro horas cada una. Al principio de la primera sesión cada profesor presentó a los estudiantes de su grupo el campo de problemas de las ecuaciones diferenciales y les propuso encontrar las soluciones analíticas de ciertas ecua. ciones a partir de la única técnica que conocían: la del cálculo de los coeficientes de la serie de Taylor de la función solución, derivando sucesivamente la ecuación y evaluando en el punto inicial. Ésta será considerada, por lo tanto, la técnica inicial del taller. Detallamos en el anexo I las pequeñas variaciones de esta técnica que debe reałizar el estudiante para llegar a dominarla.

Las limitaciones de esta técnica inicial surgen al encontrarse el estudiante con ecuaciones en las que aumenta rápidamente la dificultad del cálculo de las derivadas sucesivas y, por lo tanto, la imposibilidad de encontrar la expresión general de los coeficientes de la serie. En el anexo II se exponen las citadas limitaciones.

El hecho de centrarnos en las soluciones analíticas de las ecuaciones permite producir una nueva técnica que consiste en suponer el problema resuelto y la solución expresada como una serie de potencias; al sustituir esta serie en el lugar de la función incógnita de la ecuación diferencial, se obtiene una sucesion recurrente que permite determinar los coeficientes de la serie solución. Denominaremos esta técnica, que puede considerarse obtenida por variación de la técnica inicial, la técnica principal del taller, es decir la técnica que los estudiantes deben conseguir dominar, rutinizar y adaptar, por variación, a las nuevas situaciones problemáticas que se les presentarán. Figuran también en el anexo III el proceso de producción de la nueva técnica, la correspondiente ampliación del campo de problemas y las necesidades teóricas que ello comporta.

Al intentar rutinizar esta nueva técnica, los estudiantes toparon con numerosas dificultades que tuvieron que superar gradualmente: el cambio de variable o de super y subíndice en la serie solución; la obtención de los 
primeros términos de la serie y de la relación de recurrencia; y, sobre todo, la resolución de esta recurrencia (simple, pero poco familiar). Éstas son, a grandes rasgos, las subtécnicas de que se compone la técnica principal.

La segunda sesión tenía por objetivo utilizar la técnica principal del taller para realizar un estudio profundizado de la clase de problemas de las ecuaciones diferenciales lineales de primer orden con coeficientes constantes y con una función polinómica como término independienie. Esto permitió a los estudiantes conseguir un dominio suficientemente robusto de la técnica principal del taller para poderla modificar, estudiar el caso general presentado y conjeturar algún resultado teórico interesante que requerirá una justificación posterior (en la clase de teorf́a). En el anexo IV figura un esquema de dicho estudio profundizado.

El objetivo de la tercera sesión consistió en explorar la clase de problemas cuya incógnita es el desarrollo de Taylor de una función elemental dada, mediante una nueva modificación de la técnica principal del taller. La modificación consiste en lo siguiente: dada una función, se busca una ecuación funcional (diferencial o no) que acepte dicha función como solución; utilizando la unicidad (bajo condiciones iniciales dadas) de la supuesta solución analítica de la ecuación funcional considerada, puede entonces aplicarse la técnica principal del taller para obtener los coeficientes de la serie de Taylor buscada. (Ver los detalles en el anexo V).

Entre las posibles direcciones de desarrollo de las técnicas, complementarias de las que se han realizado en el taller, podemos citar las que llevan al estudio de las ecuaciones diferenciales de orden superior y las que ponen de manifiesto las limitaciones de la técnica principal del taller cuando se intenta resolver con ella ecuaciones diferenciales no lineales. (Ver anexo VI.)

El estudio relativamente profundizado que se ha llevado a cabo para algunas clases de problemas (dentro del campo virtual inicial) ha puesto de maniffesto, a raíz de las necesidades teóricas que se han generado, hasta qué punto el discurso teórico (jerarquizador, estructurador y justificador de los objetos matemáticos y de su manipulación) depende estrechamente, en cada momento, de las técnicas utilizadas ${ }^{12}$.

Ambas cuestiones, las posibles direcciones de desarrollo de las técnicas y la estrecha dependencia entre el discurso teórico y las técnicas utilizadas, enmarcan el ámbito del conocimiento matemático en el que un «experto» en las técnicas de este taller podría desarrollar una actividad matemática autónoma y creativa. Se pone de manifiesto, asimismo, la importancia del momento de la técnica como momento integrador del proceso de estudio de un campo de problemas.

\section{Algunos fenómenos emergentes}

Citaremos esquemáticamente algunos hechos y fenómenos didácticos que han ido emergiendo a lo largo de las experimentaciones de los talleres de prácticas que hemos realizado. Se incluyen aquí los dos talleres realizados durante los cursos 91-92 ya citados, junto al que se Ilevo a cabo durante el curso $90-91$ con estudiantes de segundo curso de licenciatura de matemáticas (Gascón 1991b):

a) Se observa una importante ralentización del cambio de los objetos matemáticos manipulados en las sesiones de prácticas, respecto a los restantes dispositivos de enseñanza (clases de teoría y clases de problemas): parece entonces, en un primer momento, que los estudiantes pasan "mucho tiempo» realizando una (en apariencia) "misma actividad» y, por tanto, que progresan muy lentamente.

b) Se ha visto también que los estudiantes, como actores principales del taller, aceptan mejor que el propio profesor de prácticas el llevar a cabo de manera «oficial» el trabajo de la técnica, seguramente porque en sus manos la utilización reiterada de una técnica que no dominan tarda más en aparecer como un trabajo rutinario. Dicho de otro modo, la ralentización a la que nos referíamos no tiene un carácter objetivo: el principiante «ve» la noveđad allí đonde el experto observador sólo vistumbra la utiłlización de una «misma técnica» en dos «problemas equivalentes».

c) Se pone claramente de relieve en los talleres de prácticas que la actividad matemática que el profesor enseña no debe ser una «copia» de la que êl mismo llevaría a cabo para estudiar el campo de problemas abordado e, incluso, que el profesor tiene a veces que exigir del estudiante un dominio de algunas técnicas localmente más robusto đel que él mismo posee: el estudiante puede tener la necesidad de llegar a ser más experto que el propio profesor por lo que se refiere a la manipulación de las técnicas del taller.

d) La institucionalización de los talleres de prácticas pone de manifiesto la necesidad imperiosa de un trabajo de ingeniería matemática, elemento esencial para la ingeniería didáctica, totalmente diferente del que hace normalmente el matemático, tanto en la investigación como en la preparación de las clases. Se trata del trabajo matemático previo a la elaboración del «material de prácticas».

e) Las dificultades encontradas para hacer vivir el momento de la técnica dentro del sistema de enseñanza de las matemáticas no provienen únicamente de la peyoracíon cultural del trabajo de la técnica. Hemos constatado además que el momento exploratorio (considerado cada vez más como el prototipo de actividad matemática por excelencia, incluso a nivel universitario) tiende a invadir el momento de la técnica. Esta «invasión» se manifiesta dentro del propio taller de prácticas y, sobre todo, fuera del mismo. Dentro del taller, en el desarrollo de cada sesión de prácticas, hay que luchar contra la tendencia escolar que lievaría a explorar constantemente problemas nuevos con técnicas nuevas y, por tanto, a minimizar el trabajo de la técnica. Desde fuera, en el diseño del proceso global de enseñanza de las matemáticas (en el caso de un nuevo plan de estudios, por ejemplo), la 
citada invasión puede manifestarse al no verse la necesidad de distinguir entre las clases de problemas y las prácticas matemáticas, lo que significa, en el fondo, negarle una identidad (esto es, una estructura y funciones propias) a la nueva actividad docente. Pensamos que la tendencia del momento exploratorio a invadir la totalidad đel proceso didáctico (tendencia dominante en la enseñanza secundaria pero, como hemos dicho, no ajena a la enseñanza universitaria) dificultará la instauración de los talleres de prácticas, perpetuando así muchas de las disfunciones observables en los actuales sistemas de enseffanza de las matemáticas.

\section{NOTAS}

' La ingeniería didáctica consiste en producir situaciones o medios de enseñanza que respondan a problemas técnicos predeterminados [...] donde los efectos se prevén o calculan en función de conocimientos precisos [de didáctica de las matemáticas]» (Brousseau 1989).

${ }^{2}$ Para más detalles sobre el estado actual de desarrollo de este modelo y su conexion con nuestro trabajo, remitimos a Chevallard 1991 y 1992, Bosch y Gascón 1992 y 1993, Gascón 1991a y 1992.

${ }^{3}$ Los desarrollos presentados en este apartado y el siguiente han sido elaborados a partir de los trabajos de Yves Chevallard citados en la nota anterior.

${ }^{4} \mathrm{Al}$ nivel de análisis en que nos situamos, tomaremos como primitivas las nociones de "problema», «campo de problemas», «técnica matemática» y «teoría». Aunque, en otro nivel de análisis, es posible preguntarse, por ejemplo, cuáles son los componentes de una técnica matemática o de un campo de problemas, aquí sólo mostraremos la forma de empleo de estas nociones y sus interrelaciones.

${ }^{5}$ Nuestro postulado, al identificar la actividad matemática con la utilización y producción de técnicas matemáticas, es una especificación de un postulado antropológico general según el cual toda actividad humana resulta de la puesta en práctica de una técnica (notaremos pues que la noción de «técnica» tiene aquí un sentido mucho más amplio todavía).

${ }^{6}$ Veáse Kuhn 1962, Lakatos, 1971 1976, 1978, Feyerabend 1970, Toulmin 1972 y Serres 1989

${ }^{7}$ En el caso de la última reforma del currículo de matemáticas de secundaria, aparece una tendencia a reemplazar la práctica de la actividad matemática por la enseñanza de una supuesta "cultura matemática» que forzosamente quedará vacía sỉ no se fundamenta en el dominio y desarrollo de las técnicas matemáticas. Este fenómeno, que se extiende a otras disciplinas, fue notado por Agustín García Calvo, quien interpreta que la tendencia de ir reemplazando sistemáticamente la enseñanza del griego y el latín por una «cultura clásica» en los (sucesivos) "nuevos» planes de estudio se apoya en una idea dominante (falsa, como todas) respecto a las relaciones entre lenguaje y cultura, idea que atribuye a esta última una importancia desmesurada y paradojica al presentarla como independiente de la actividad que la genera (el estudio de las lenguas clásicas en este caso) (García Calvo 1992).

${ }^{8}$ Esta característica es común al experto en cualquier tipo de técnicas; ya sea en técnicas aprendidas de manera no consciente
Las descripciones anteriores, forzosamente esquemáticas y desligadas, no pretenden en ningún modo astumir el papel de análisis o evaluación prematura de la actividad docente experimentada. Es previsible que el estudio sistemático de estos fenómenos sobrepase ampliamente el ámbito del taller de prácticas para abordar de lleno las interrelaciones de éste con los restantes dispositivos del sistema de enseñanza de las matemáticas. Este estudio se situará, por tanto, en el marco de un nuevo programa de investigación en didáctica de las matemáticas en el que debemos seguir trabajando ${ }^{13}$.

(como ciertas técnicas del equilibrio, las técnicas que nos permiten discriminar las oraciones «bien formadas» en nuestra Iengua materna o las técricas de la orientación espacial $\rangle$, como en técnicas aprendidas de manera más consciente (como las técnicas del juego del ajedrez, las que permiten tocar un instrumento musical o las técnicas matemáticas institucionalizadas).

${ }^{9}$ A fin de mostrar más claramente este fenómeno, diremos que la represión del trabajo de la técnica no tiene el mismo alcance en las actividades llamadas "manuales", en las actividades deportivas y ni siquiera en las artísticas donde, aunque siempre se considera que la técnica debe estar al "servicio» de la creatividad, no deja de verse como una condición indispensable de ésta.

${ }^{10}$ Esta microprueba es un dispositivo didáctico importante debido a su función de objetivización, puesto que da al estudiante una valoración externa de los resultados del trabajo técnico realizado. También ayuda a definir el «contrato didáctico» que se establece a lo largo del taller, al precisar en particular hasta qué punto los estudiantes deben dominar las técnicas utilizadas en cada sesion para proseguir con el estudio del campo de problemas. No es necesario decir que la microprueba también funciona como una importante fuente de información para los profesores ( $y$, en este caso, para los investigadores).

${ }^{11}$ El hecho de haber iniciado la experimentación con estudiantes universitarios es meramente circunstancial: el lector habrá podido observar que el alcance de nuestras formulaciones no depende del nivel educativo considerado. Se verá asimismo que el trabajo de ingeniería realizado se podría extender (con algunas modificaciones) a los niveles preuniversitarios.

${ }^{12}$ Asf́, por ejemplo, durante la utilización de la técrica inicial del taller, puede surgir la necesidad de relacionar la unicidad de la solución de las ecuaciones diferenciales estudiadas con la de las relaciones de recurrencia obtenidas, así como con la unicidad de la serie de Taylor de una función analítica; puede aparecer astmismo la necesidad de justificar algunas de las manipulaciones (como los cambios de variable de las series de potencias), la de asegurar la convergencia de la serie encontrada; etcétera.

${ }^{13}$ Agradecemos a G. Brousseau, Y. Chevallard, P. Orús y J.M. Lamarca Ia lectura de las versiones preliminares de este trabajo, así como sus sugerencias para mejorarlo. Para orientación del lector, queremos hacer constar que en este artículo se describe la teoría de los momentos didácticos y su experimentación en el estado en que se encontraban en abril de 1992, fecha en la que fue elaborado este trabajo. 


\section{REFERENCIAS BIBLIOGRÁFICAS}

BOSCH, M., 1992. Sèries de potències i equacions diferencials, material de prácticas de Análisis I. (Documento interno no publicado.)

BOSCH, M. y GASCÓN, J., 1992. Una nova activitat docent: les pràctiques a la llicenciatura de matemàtiques, comunicacion presentada en las Jornades sobre innovació en la docència universitària, Univiversitat Autònoma de Barcelona, abril de 1992.

BOSCH, M. y GASCÓN, J, 1993. Prácticas en matemáticas: el trabajo de la técnica, en Filloy, Puig y Rojano, (eds.), Historia de las ideas algebraicas, Memorias del Tercer Simposio Intermacional sobre Investigación en Educación Matemática. Valencia, junio 1991, pp. 141-151.

BROUSSEAU, G., 1981. Problèmes de didactique des décimaux, Recherches en Didactique des Mathématiques, 2.3, pp. $37-127$.

BROUSSEAU, G., 1986. Fondements et méthodes de la didactique des mathematiques, Recherches en Didactique des Mathématigues, 7.2, pp. 37+115.

BROUSSEAU, G., 1989. La tour de Babel, Etudes en Didactique des Mathématiques, article occasionnel n. 2, IREM de Bordeaux,

CHEVALLARD, Y., 1991. Un problema de ingeniería didáctica de los sistemas de formación: las prácticas en matemáticas, curso impartido en el Seminari de Didàctica de les Matemàtiques del Departament de Matemàtiques de la UAB, febrero de 1991.

CHEVALLARD, Y., 1992. Hacia una teoría de los momentos didácticos, curso de doctorado de Didáctica de las Matemáticas impartido en la Universidad de Granada, febrero de 1992.

FEYERABEND,P.K., 1970.Against Method, MinnesotaStudies in the Philosophy of Science, vol. IV. (University of Minnesota: Minneapolis). Trad.: 1974, Contra el método. (Ariel: Barcelona).

GARCÍA CALVO, A., 1992. Recuerdo de la charla dada en Alcalá de Henares el 6 de abril de 1989, en Gómez, F.J. y Gómez-Pantoja, J., (eds.), Pautas para una seducción. Ideas y materiales para una nueva asignatura: Cultura Clásica. (ICE de la Universidad de Alcalá de Henares: Madrid).

GASCON, J., 199 la. Anàlisi de les funcions de les pràctiques en els actuals sistemes d'estudi: el cas de la licenciatura de matemàtiques, Curso impartido en el Seminari de Didàctica de les Matemàtiques del Departament de Matemàtiques de la $\mathrm{UAB}$.

GASCÓN, J., 1991b. Desenvolupament i producció d'algunes tècniques d'estudi del camp de problemes de les successions recurrents, Curso impartido en el Seminari de Didàctica de les Matemàtiques del Departament de Matemàtiques de la UAB.

GASCÓN, J., 1992. Què s'entén per Resolució de Problemes de Matemàtiques?, Biaix, 2, pp. 10-17.

GASCON, J., 1993. Desarrollo del conocimiento matemático y análisis didáctico: del patrón de análisis-síntesis a la génesis del lenguaje algebraico, Recherches en Didactique des Mathématiques, 13.3,pp. 295-332.

KUHN, T.S., 1962. The structure of Scientific Revolutions, 2a ed. (University Chicago Press: Chicago). Trad.: 1971, La estructura de las revoluciones cientificas. (Fondo de Cultura Económica: México D.F.).

LAKATOS, I., 1971, History of Science and its Rational Reconstructions, en R.C. Buck y R.S.Coten (eds.), PSA 1970. Boston Studies en the Philosophy of Science, 8, (Reidel: Dordrecht) pp. 91-135. Trad.: 1974, Historia de las ciencias y sus reconstrucciones racionales. (Tecnos: Madrid).

LAKATOS, I., 1976. Proofs and Refutations. The logic of Mathematical Discovery. (Cambridge University Press: Cambridge). Trad.: 1978, Pruebas y refutaciones. La lógica del descubrimiento matemático. (Alianza: Madrid).

LAKATOS, I., 1978. Mathematics, science and epistemology. Philosophical Papers, Vol. 2, editado por Worrall, J. y Currie, G. (Cambridge University Press: Cambridge). Trad.: 1981, Matemáticas, ciencia yepistemologia, Vol 2. (Alianza: Madrid).

SERRES, M., 1989. Eléments d'Histoire des Sciences. (Bordas: París). Trad.: 1991, Historia de las ciencias. (Cátedra: Madrid).

TOULMIN, S., 1972. Human understanding, Vol.1. (Princeton University Press). Trad.: 1977, La comprension humana. Vol. 1. El uso colectivo y la evolución de los conceptos. (Alianza: Madrid).

WHITEHEAD, A.N., 1929. The Aims of Education, and Other Essays. Trad.: 1957, Los fines de la Educacion y otros ensayos. (Paidos: Buenos Aires). 


\section{ANEXO I, TÉCNICA INICIAL}

El estudio del campo de problemas se empieza intentando hallar las soluciones analiticas de ciertas ecuaciones diferenciales lineales. La técrica inicial consiste b6sioamente en calcular, a partir de la ecuación diferencial, les derivadas sucesivas de la función sohución pare deducir su serie de Taylor e intentar deopués identificarla en términos de funciones elementales.

(1) $\left\{\begin{array}{l}y^{\prime}=-3 y \\ y(0)=14\end{array}\right.$

Observaciones previas sobre el groblema:

Sea $y(x)$ una solución de la ecuación. La forma misma de la ecuación nos permite afirmar que toda solución será infinitamente derivable en su dominio de definición: y es una vez derivable $y$, puesto que se cumple $y^{\prime}=-3 y, y^{\prime}$ también será una vez derivable, etc.

Consideraremos unicamente las soluciones analáticas de la ecuación, expresadas mediante su serie de Taylor. Como toda solución verifica $y(0)=14$, consideraremos la serie de Taylor centrada en $x=0$ :

$$
y(x)=\sum_{n \geq 0} \frac{y^{(n)}(0)}{n !} x^{n}
$$

Determinación de los términos de la serie solsción:

Sustituimos $y(x)$ por su serie de Taylor en la ecuación y derivamos esta sucesivamente:

$$
\begin{aligned}
& y(0)=14 \\
& y^{\prime}=-3 y \quad \Longrightarrow \quad y^{\prime}(0)=-3 y(0)=-3 \times 14 \\
& y^{\prime \prime}=-3 y^{\prime}=(-3)^{2} y \Longrightarrow y^{\prime \prime}(0)=(-3)^{2} 14 \\
& y^{(n)}=-3 y^{(n-1)}=(-3)^{n} y \Rightarrow y^{(n)}(0)=(-3)^{n} 14
\end{aligned}
$$

Conclusión: la ecuación tiene una única solución analítica:

$$
y(x)=\sum_{n \geq 0} \frac{(-3)^{n} 14}{n !} x^{n}=14 \sum_{n \geq 0} \frac{(-3)^{n}}{n !} x^{n}
$$

Radio de convergencia e identificación de la serie obtenida:

El calculo del radio de convergencia nos determina el dominio de definición de la solución;

$$
R^{-1}=\lim _{n \rightarrow \infty} \frac{(-3)^{n+1} n !}{(-3)^{n}(n+1) !}=\lim _{n \rightarrow \infty} \frac{(-3)}{n+1}=0 \Longrightarrow R=\infty
$$

La solución esté definida para todo $x \in \mathbf{R}$.

La igualdad $y(x)=14 \sum \frac{(-3)^{n}}{n} x^{n}=14 \sum \frac{(-3 x)^{n}}{n \mid}$ permite identificar la solución como la serie de Taylor de la función $y(x)=14 e^{-3 x}$.

Sabemos, por otra parte, que esta función está definida para todo $x \in R$, lo que concuerda con el resultado anterior.

Comprobación: La solución obtenida es:

$$
y(x)=14 e^{-3 x}
$$

que satisface efectivamente la ecuacion: $\left(14 e^{-3 x}\right)^{\prime}=-3\left(14 e^{-3 x}\right)$, y la condición injcial: $14 e^{0}=14$.

Ampliación del problema:

Notemos que la condición inicial $y(0)=14$ determina los coeficientes de la serie $y$, por tanto, la unicidad de la solución. Con una condicion inicial general $y(0)=y_{0} \in \mathbf{R}$, obtendrfamos, para todo $n, y^{n}(0)=(-3)^{n} y_{0}$, lo que nos da la solución general: 


$$
y(x)=\sum_{n \geq 0} \frac{(-3)^{n} 30}{n !} x^{n}=30 e^{-3 x}
$$

Así, la tunción $y(x)=y 0 e^{-9 x}$ es la única solución analítice de la ecuación diferencial $y^{\prime}=-3 y$ con $y(0)=y 0$.

En caso de tener una condición infcial del tipo $y\left(x_{0}\right)=y_{0}$ (con $x_{0}$ no necesariamente igual a $0)$, buscarfamos una solución de la forma $y(x)=\sum_{n \geq 0} \frac{y^{(n)}\left(x_{0}\right)}{n !}\left(x-x_{0}\right)^{n}$.

Si consideramos ahora la ecuación general $y^{\prime}=\alpha y$ con $\alpha \in R$, entonces la derivada $n$-ésima de la solución será $y^{(n)}\left(x_{0}\right)=\alpha^{n} y_{0}$, y la serie solución:

$$
y(x)=y_{0} \sum_{n \geq 0} \frac{\alpha^{n}}{n !}\left(x-x_{0}\right)^{n}
$$

que corresponde a la función $y(x)=y_{0} e^{\alpha\left(x-x_{0}\right)}$.

(2) $\left\{\begin{array}{l}y^{\prime}=5 y+x \\ y(0)=1\end{array}\right.$

\section{Observaciones previos:}

A diferencia de la ecuación anterior, aparece aquf una $x$ como segundo sumando en la ecusción (esto es, aparece un término independiente). Este término independiente "invalida" una posible solucion del tipo $y(x)=y_{0} e^{a x}$.

Esta novedad del problema no trae consecuencias mayores, solo hace más compleja la utilización de la técnica (sobre todo en la identificación de la solución).

Serie de Taylor de $y(x)$ en torno a $x=0: y(x)=\sum_{n \geq 0} \frac{y^{(n)}(0)}{n j} x^{n}$

Determinación de los términos de la serie solución:

$$
\begin{aligned}
& y(0)=1 \\
& y^{\prime}=5 y+x \quad \Longrightarrow y^{\prime}(0)=5 y(0)=5 \\
& y^{\prime \prime}=5 y^{\prime}+1 \Longrightarrow y^{\prime \prime}(0)=25+1=26 \\
& y^{\prime \prime \prime}=5 y^{\prime \prime} \Longrightarrow y^{\prime \prime \prime}(0)=5 \times 26 \\
& y^{\prime \prime \prime}=5 y^{\prime \prime \prime}=5^{2} y^{\prime \prime} \quad \Longrightarrow \quad y^{\prime \prime \prime}(0)=5^{2} \times 26 \\
& y^{(n)}=5 y^{(n-1)} \Rightarrow 5^{n-2} y^{\prime \prime} \Rightarrow y^{(n)}(0)=5^{n-2} y^{\prime \prime}(0)=5^{n-2} 26 \quad \forall n \geq 2
\end{aligned}
$$

Conclusión: La ecuación tiene uns únjca solución snalítica:

$$
y(x)=1+5 x+\sum_{n \geq 2} \frac{5^{n-2} 26}{n !} x^{n}=1+5 x+26 \sum_{n \geq 2} \frac{5^{n-2}}{n !} x^{n}
$$

Para hacer aparecer en la expresión obtenida alguna serie de Taylor conocida, debemos entonces realizar el siguiente trabajo:

$1+5 x+26 \sum_{n \geq 2} \frac{5^{n-2}}{n !} x^{n}=1+5 x+\frac{26}{25} \sum_{n \geq 2} \frac{5^{n}}{n !} x^{n}=1+5 x+\frac{26}{25}\left(\sum_{n \geq 0} \frac{5^{n}}{n !} x^{n}-1-5 x\right)=\frac{26}{25} e^{5 x}-\frac{1}{25}-\frac{1}{5} x$

La solución es pues: $y(x)=\frac{26}{25} e^{5 x}-\frac{1}{25}-\frac{1}{5} x$ definida para todo $x$ real.

Vemos omo la ldentificación de la serie obtenida en terminos de funciones elementales determina el dominio de defintción de la solución y hace innecesario el cálculo del radio de convergencia. 


\section{Dificultades técricas encontradas:}

La "novedad" del término independiente $(\neq 0)$ de la ecuacion no nos ha impedido encontrar la expresión general de la derivada nésima de la solución puesto que dicho têrmino desaparece al derivar sucesivamente la ecuacion. (No consideraremos aqui las importantes variactones técnicas que serfan necesariss si se quisieran tesolver ecuaciones diferenciales lineales con tórminos independientes no polinómicos.)

Nótese quo la identfficación de la solución results en este caso de la utilización de una subtécnios de la técrion inicial con un "modo de funcionamiento" propio (y nade trivial): cambios de sublndices, introducción y substracción de términos, etc.

\section{Ampliación del problema:}

El estudio que acabarnos de llevar a cabo con la ecuación $y^{\prime}=5 y+x$ se puede generalizar para ecuaciones del tipo $y^{\prime}=\infty y+x, \operatorname{con} \alpha \in R$, y condición inicial $y\left(y_{0}\right)=x_{0}$.

Los mismos "gestos" efectuados anteriormente permiten llegar a le solución:

$$
y(x)=y_{0}+\alpha y_{0}\left(x-x_{0}\right)+\left(\alpha^{2} y_{0}+1\right) \sum_{n \geq 2} \frac{a^{n-2}}{n !}\left(x-x_{0}\right)^{n}
$$

que sólo babría que identificar en términos de funciones elementales.

Es fácil ver que las pequeñas variaciones de la técnice utilizsda para resolver $y^{f}=a y+x$ nos permitirfan proseguir con un estudio "sistemático" de las ecusciones diferencieles lineales de primer orden con coeficientes constantes y término independiente polinómico:

$$
y^{\prime}=\alpha y+P(x) \text {. }
$$

Dado que, para $n$ suficientemente grande, siempre obtendremos ls igualdad $y^{(n)}\left(x_{0}\right)=\alpha y^{(n-1)}$, podemos asegurar que en la función solución aparecerá el término $e^{\alpha\left(x-x_{0}\right)}$ (esto la solución de la ecuactón lineal homogenea $y^{\prime}=\alpha y$ asociada a $y^{\prime}=\alpha y+P(x)$ ).

El trabajo de la técnica seguiría con el examen de las siguientes ectaciones (que dejamos al lector):

(3) $\left\{\begin{array}{l}y^{\prime}=y+2 x^{2} \\ y(1)=0\end{array}\right.$

(4) $\left\{\begin{array}{l}y^{\prime}=x y \\ y(0)=1\end{array}\right.$

Podemos quizs prever en este altimo caso que la aparición de coeficientes no constantes hago la utilización de la técnica más "sensible" a pequexãas variaciones de la ecuación, pudiéndose presentar aqus una verdadera limitación de la técnica. 
(6) $\left\{\begin{array}{l}y^{\prime}+3 x^{2} y-x^{2}=0 \\ y(0)=0\end{array}\right.$

\section{Observaciones previos:}

Antes de resolver la ecuación, notemos que ésta no aparece en la forms hasts ahora habitual $y^{\prime}=F(y, x)$, sino en la forma $G\left(x, y, y^{\prime}\right)=0$. Para mayor comodidad la transformaremos en la ecusacion equivalente

$$
y^{\prime}=-3 x^{2} y+x^{2}
$$

También podemos notar que el coeficiente de lo y no es constante, lo que podrá dificultar el cálculo de las derivadss sucesivas. La condición inicial en cambio aparece en su "versión simple", evaluada en $x=0$. Buscamos por lo tanto una solución de la forma:

$$
y(x)=\sum_{n \geq 0} \frac{y^{(n)}(0)}{n !} x^{n}
$$

Determinación de los términos de la serie:

$$
\begin{aligned}
y^{\prime}=-3 x^{2} y+x^{2} & \Longrightarrow y^{\prime}(0)=0 \\
y^{\prime \prime}=-6 x y-3 x^{2} y^{\prime}+2 x & \Longrightarrow y^{\prime \prime}(0)=0 \\
y^{\prime \prime \prime}=-6 y-12 x y^{\prime}-3 x^{2} y^{\prime \prime}+2 & \Longrightarrow y^{\prime \prime \prime}(0)=2 \\
y^{\prime \prime \prime \prime}=-18 y^{\prime}-18 x y^{\prime \prime}-3 x^{2} y^{\prime \prime \prime} & \Longrightarrow y^{\prime \prime \prime}(0)=0 \\
y^{(5)}=-36 y^{\prime \prime}-24 x y^{\prime \prime \prime}-3 x^{2} y^{\prime \prime \prime} & \Longrightarrow y^{(5)}(0)=0 \\
y^{(6)}=-60 y^{\prime \prime \prime}-30 x y^{\prime \prime \prime}-3 x^{2} y^{(5)} & \Longrightarrow y^{(6)}(0)=-60 \times 2 \\
y^{(7)}=-90 y^{\prime \prime \prime}-36 x y^{(5)}-3 x^{2} y^{(6)} & \Longrightarrow y^{(7)}(0)=0 \\
y^{(8)}=-125 y^{(5)}-42 x y^{(6)}-3 x^{2} y^{(7)} & \Longrightarrow y^{(8)}(0)=0 \\
y^{(9)}=-168 y^{(6)}-48 x y^{(7)}-3 x^{2} y^{(8)} & \Longrightarrow y^{(9)}(0)=168 \times 60 \times 2 \\
\cdots & \cdots \\
y^{(n)}=? & \Longrightarrow y^{(n)}(0)=?
\end{aligned}
$$

\section{Dificultadea técricas:}

Serfa muy largo y penoso seguit derivando la solución para intentar hallar el término general de Is derivads $n$-nésima. Lo único que se ve, de momento, que $y^{(n)}=0$ ai $n$ no es multiplo de 3 , caracteristica sta que no depende de la condición inicial dada.

También podemos afirmar que si la condición inicial es tal que $y^{\prime \prime \prime}(0)=0$ (esto ocurrirfa si tuviéramos $y(0)=\frac{1}{3}$ ), entonces la única solución de la ecuación es la función constante $y=\frac{1}{3}$. Por contra, con una condición inicial en $x_{0} \neq 0$, aún sería más diffcil encontrar una expresión pars $y^{(n)}(0)$.

En resumen, la técnica inicial nos permite resolver ecuaciones diferenciales lineales de primer onden con coeficientes constentes, pero no todas las ecusciones con coeficientes polinbmicos de grado mayor que 1. Las limitaciones de esta técnica inicial provienen de las dificultades para hallar la expresión generul de $y^{(n)}(0)$ (y pues de los coeficientes de lo serie solución). 


\section{ANEXO III. PRODUCCIÓN DE UNA NUEVA TÉCNICA}

Intentaremos solventar la limüación de la técnica inicial iniroduciendo la siguiente variación: dada una ecuación diferencial de primer orden $F\left(y^{\prime}, y, x\right)=0$, supongamos que el problema de hallar una solución analitios yo esta resuelto y expresemos dicho solución en forma de serie de potencias: $y(x)=\sum a_{n} x^{n}$. Tenemas entonces que $y^{\prime}(x)=\sum \operatorname{ran}_{n} x^{n-1}$.

Si rustituimos en lo ecuación y e $y^{\prime}$ por sts exprestón en serie de potencios, la ecuación se convertió en una igualdad de series. Igtualando los coeficientes de las series, podemos deducir una relación de recurrencia entre los $a_{n}$. La solución de esta relación es lo que nos permitirí determinar la función solución.

Podemos considerar que esta madificación no constiluye t́nicamente una varisclón de la técnion inicicl, sino que da lugar a tue nueva técnica de estudio de las ecuaciones diferenciales lineales: la llamaremos la técnica principal del taller. Con ella, el "trabajo" de resolusción de las ecuaciones diferenciales se va a transformar completamente, como veremos a continuación retomando las primeras ectaciones estudiadas anteriomente.

(1) $\left\{\begin{array}{l}y^{\prime}=-3 y \\ y(0)=14\end{array}\right.$

Sustitución de in solución por su desarmilio en serie:

Sea $y(x)=\sum_{n \geq 0} a_{n} x^{n}$ la solución expresada con su desarrollo en serie de potencias (centrada en $x=0$ porque la condición inicial viene dada en este punto).

Si sustituimos en la ecuación tenemos:

$$
\begin{aligned}
y^{\prime}(x)=-3 y(x) & \Leftrightarrow \sum_{n \geq 1} n a_{n} x^{n+2}=-3 \sum_{n \geq 0} a_{n} x^{n} \\
& \Leftrightarrow \sum_{n \geq 0}(n+1) a_{n+1} x^{n}=\sum_{n \geq 0}-3 a_{n} x^{n} \\
& \Leftrightarrow(n+1) a_{n+1}=-3 a_{n} \quad \forall n \geq 0
\end{aligned}
$$

Deteminación de los coeficientes de la serie:

Obtenemos asf una relocion de recurrencia entre los coeficientes de la sarfe solución:

$$
a_{n+1}=\frac{-3}{n+1} a_{n} \quad \text { para todo } n \geq 0
$$

que nos permitiré haller la expresión del término general:

$$
a_{1}=-3 a_{0} ; \quad a_{2}=\frac{(-3)^{2}}{2} a_{0} ; \quad \ldots \quad a_{n}=\frac{-3}{n} a_{n-1}=\frac{-3}{n} \frac{-3}{n-1} \ldots \frac{-3}{2} a_{0}=\frac{(-3)^{n}}{n !} a_{0}
$$

Deducimos que $a_{n}=\frac{(-3)^{n}}{n !} a_{0}$ para todo $n \geq 0$ con $a_{0}=y(0)=14$ : Obtenemos entonces el término general:

Identificación de la solucion:

La ecuación tiene una única solución analítics:

$$
y(x)=\sum_{n \geq 0} \frac{(-3)^{n}}{n !} 14 x^{n}=14 \sum_{n \geq 0} \frac{1}{n !}(-3 x)^{n}=14 e^{-8 x}
$$

Dificultades técnicas:

En el estudio de este tipo de ecurción, la nueva técnica se muestra menos económica que la inicial. Esto quizá debido a la novedad de su utilización y a una posible inexperiencia del actor en la identificación de sucesiones recurrentes. Es de prever que, a medida que se avance en el trabajo de la técnica, estas pequeñas dificultades disminuysn paulatinamente y nos permitan realizar pequeñas variaciones para solventar los problemas nuevos que puedan aparecer.

Estas nuevas dificultades técnicas son básicamente dos: la manipulación de subindices en la expresión de la serie pars facilitar la igualación de coeficientes; $y$, sobre todo, la resolución de la relación de recurrencis que se obtiene a partir de dicha igualactón. 


\section{Necesidades térices:}

Hemos utllizado implícitamente un resultado teórico importante: Ia unicidad de la expresión de una función analítica como serie de potencias. Este resultado es el que justifica el "gesto" de igualación de los oceficientes y nos asegura la obtención de una relación de recurrencia para cada ectración.

La ecuación diferencial tendrá entonces tantas soluciones analfticas como sucesiones verifiquen Ia relación de recurrencis asociada.

\section{Ampliación del problema:}

Se ve muy claramente, $\infty$ n esta técnica, que la condición inicial determina la unicidad de la solución y que, a una condición inicial $y\left(x_{0}\right)=30_{1}$ le corresponde la solucton:

$$
y(x)=\sum_{n \geq 0} \frac{y_{0}}{n !}\left(x-x_{0}\right)^{n}=z_{0} \sum_{n \geq 0} \frac{1}{n !}\left(x-x_{0}\right)^{n}=y_{0} e^{\left(x-x_{0}\right)}
$$

También podemos notar, sin necesidad de répetir todo el proceso, que a una ecuación del tipo $y^{\prime}=\alpha y$ con $\alpha \in R$ le corresponde la relación de recurrencia $a_{n}=\frac{\alpha}{n} a_{n-1}$ que determina el término general de la serie $a_{n}=\frac{a^{n}}{n} y_{0}$ y pues la solución:

$$
y(x)=\sum \frac{y_{0} \alpha^{n}}{n_{3} !}\left(x-x_{0}\right)^{n}=y_{0} \sum \frac{1}{n !}\left(\alpha\left(x-x_{0}\right)\right)^{n}=y_{0} e^{\alpha\left(x-x_{0}\right)}
$$

Posible variación de la tecrica principal:

Existe una "variante" de esta técnica que permite controlar (e incluso evitar) la manipulación de los sumstorios. Consiste en escribir las series de potencias en toda su extensión, los dos miembros de la ecuación uno encima del otro, e iguelar término a término:

$$
\begin{aligned}
& y(x)=a_{0}+a_{1} x+a_{2} x^{2}+\ldots+a_{n}+\ldots \\
& y^{\prime}(x)=a_{1}+2 a_{2} x+3 a_{3} x^{2}+\ldots+n a_{n-1}+\ldots \\
& y=y^{\prime} \Longleftrightarrow a_{0}=a_{1} ; \quad a_{1}=2 a_{2} ; \quad a_{2}=3 a_{3} ; \ldots ; \quad a_{n}=n a_{n-1} ; \ldots
\end{aligned}
$$

El trabajo de la técnica principal deberźa proseguir con la resolución de las demás ecuaciones, que no detallaremos en esta presentación. 
Los "gestos" realizados en el estudio anterior tienen un alcance mayor que la mers resolución de la ecuasión (1): parece que podemos repetir el misrno proceso con cuslquier enuación lineal oon coeficientes constantes y término independiente polinómico del tipo:

$$
y^{\prime}=\alpha y+P(x)
$$

La técnica principal utilizada hace corresponder a cada ecuación uns relsción de recurrencia, que será, en este caso, una recurrencia de primer orden. Como el término independiente de la ecuación solo afecta a los primeros coeficientes de la serie, podemos prever que, a partir de cierto $n$, obtendremos siempre una relación de recurrencia del tipo $a_{n}=a a_{n-1}$ que se puede resolver fácilmente y que dars lugar, en la solución, a un término exponenclal del tipo $e^{\alpha x}$.

Se podrá conjeturar entonces un caso particular del resultado tobrico más general según el cual las soluciones de una ecusción diferencial lineal son sumb de una ecuación particular y de la solución general de la ecuación homogénea asociada.

Resumimos a continuación los principales "gestos" del estudio de los primeros casos de esta ecuación general:

$$
\begin{aligned}
y^{\prime}=\alpha y & \Leftrightarrow \sum(n+1) a_{n+1} x^{n}=\alpha \sum a_{n} x^{n} \\
& \Leftrightarrow a_{n+1}=\frac{\alpha}{n+1} a_{n} \quad \forall n \geq 0 \\
& \Leftrightarrow a_{n}=\frac{\alpha^{n}}{n !} a_{0} \quad \forall n \geq 0 \\
& \Leftrightarrow y(x)=a_{0} \sum \frac{\alpha^{n}}{n !} x^{n}=a_{0} e^{\alpha x} \\
y^{\prime}=\alpha y+\beta & \Leftrightarrow \sum(n+1) a_{n+1} x^{n}=\alpha \sum a_{n} x^{n}+\beta \\
& \Leftrightarrow a_{1}=\alpha a_{0}+\beta ; \quad a_{n+1}=\frac{\alpha}{n+1} a_{n} \quad \forall n \geq 1 \\
& \Leftrightarrow a_{1}=\alpha a_{0}+\beta ; \quad a_{n}=\frac{\alpha^{n}}{n !}\left(a_{0}+\frac{\beta}{\alpha}\right) \quad \forall n>1 \\
& \Leftrightarrow y(x)=\left(a_{0}+\frac{\beta}{\alpha}\right) \sum_{n \geq 1} \frac{\alpha^{n}}{n !} x^{n}+a_{0}=\left(a_{0}+\frac{\beta}{\alpha}\right) e^{\alpha x}-\frac{\beta}{\alpha} \\
y^{\prime}=\alpha y+\beta x+\gamma & \Leftrightarrow \sum(n+1) a_{n+1} x^{n}=\alpha \sum a_{n} x^{n}+\beta x+\gamma \\
& \Leftrightarrow a_{1}=\alpha a_{0}+\gamma ; \quad a_{2}=\alpha a_{1}+\beta ; \quad a_{n+1}=\frac{\alpha}{n+1} a_{n} \quad \forall n \geq 2 \\
& \Leftrightarrow a_{1}=\alpha a_{0}+\gamma ; \quad a_{2}=\alpha a_{1}+\beta ; \quad a_{n}=\frac{\alpha^{n-2}}{n !} \quad \forall n>1
\end{aligned}
$$

Etc. 
Abondaremos ahora con la técrica principal la primera ecuación en lo que se pusieron claramente de manifiesto las limitaciones de la técrioa inicial.

(5) $\left\{\begin{array}{l}y^{\prime}+3 x^{2} y-x^{2}=0 \\ y(0)=0\end{array}\right.$

Sustitución de la solución por ste desartollo en serie:

Sea $y(x)=\sum_{n \geq 0} a_{n} x^{n}$ uns solución de la ecuación. Tenemos:

$$
\begin{aligned}
y^{\prime}(x)+3 x^{2} y(x)-x^{2}=0 \Longleftrightarrow \sum_{n \geq 0}(n+1) a_{n+1} x^{n}+3 \sum_{n \geq 0} a_{n} x^{n+2}-x^{2}=0 \\
\Longleftrightarrow \sum_{n \geq 0}(n+1) a_{n+1} x^{n}+3 \sum_{n \geq 2} a_{n-2} x^{n}-x^{2}=0 \\
\Longleftrightarrow \begin{array}{l}
a_{1}=0 ; \quad 2 a_{2}=0 ; \quad 3 a_{3}+3 a_{0}-1=0 ; \\
(n+1) a_{n+1}+3 a_{n-2}=0 \quad \forall n \geq 3
\end{array}
\end{aligned}
$$

Relación de recurrencia:

Obtenemos esta vez una relación de recurrencia de orden 3:

$$
a_{0}=a_{0} ; \quad a_{1}=0 ; \quad a_{2}=0 ; \quad a_{3}=\frac{1}{3}\left(-3 a_{0}+1\right) ; \quad a_{n}=\frac{-3}{n} a_{n-3} \quad \forall n \geq 4
$$

Resolución de la recturnencia:

$$
a_{0,} a_{1}, a_{2}=0 ; \quad a_{3}=\frac{1}{3}\left(1-3 a_{0}\right) ; \quad a_{4}=0 ; \ldots ; a_{n}= \begin{cases}\frac{-3}{n} \frac{-9}{n-3} \ldots \frac{-3}{6} a_{3} & \text { ti } n=3 k \\ 0 \text { si no } & \end{cases}
$$

Identificación de la solución:

Los términos no nulos de la serie son:

$$
a_{3 k}=\frac{(-3)^{k-1}}{3^{k-1} k !} a_{3}=\frac{(-1)^{k-1}}{3 k !}\left(1-3 a_{0}\right) \quad \forall k \geq 0
$$

Para $a_{0}=y(0)=0$, la ecuación tiene una única solución analítica:

$$
y(x)=\sum_{k \geq 0} \frac{(-1)^{k-1}}{3 k !} x^{3 k}
$$

que identificamos como $y(x)=-\frac{1}{3}\left(\exp \left(-x^{3}\right)+1\right)$.

Ampliación del problema:

La solución de la ecuación $y^{\prime}+3 x^{2} y-x^{2}=0$ con condición inictal $y\left(x_{0}\right)=a_{0}$ es:

$$
y(x)=\left(a_{0}-\frac{1}{3}\right) \exp \left(-\left(x-x_{0}\right)^{3}\right)
$$

Vemos que con la condición inicial $y\left(x_{0}\right)=\frac{1}{3}$ la solución es $y(x) \equiv 0$. (Formaris parte del trabajo de la ténica el volver a resolver la ecuación en este caso concreto para comprobar que ae obtiene efectivamente este resultado (ya) conocido.)

La tecnica prinaipal nos ha permitido resolver una ecuación con la que habta fracasado la técnioa inicial. Pero esta mejorn se ha hecho bajo ciertas restricciones, puesto que no "controlamos" todavia el alcance de la nueva técnica. Necesitartamos grofundizar mas an el estudio pare pare delimitar la clase de ecuaciones diferenciales que la técricos prinicipal nos permite estudiar (aber cuclies son los tipos de recurrencias que nos pueden aparecer, si son o no resolubles, etc.). 
La téenio principal no nos permite tinicamente resolver ecuaciones diferenciales lineales. Con una nueva variación, se puede ampliar el campo de problemas estudiado, no pare resolver ecuaciones diferenciales nuevas, sino para encontrar la serie de Taylor de una función elemental dada, hallando en primer lugar una ecuación diferencial o funcional que la tenga como única solución.

Ejomplo: Queremos hallar la serie de Taylor de la función $f(x)=\sqrt{x+1}$.

$$
f(x)=\sqrt{x+1} \Longrightarrow f^{\prime}(x)=\frac{1}{2 \sqrt{x+1}} \Rightarrow 2(x+1) f^{\prime}(x)=f(x)
$$

Por lo tanto $f(x)$ es solución de la ecuación diferencial lineal $2(x+1) y^{\prime}=y$.

Resolvemos esta ecuación con la técnica principal y obtenemos:

$$
2(x+1) \sum_{n \geq 0} n a_{n} x^{n-1}=\sum_{n \geq 0} a_{n} x^{n} \Leftrightarrow a_{n+1}=\frac{1-2 n}{n+1} a_{n} \quad \forall n \geq 0
$$

Añadiendo la condición inicial $f(0)=1$, teremos que $a_{0}=1$ y pues:

$$
f(x)=1+\frac{1}{2} x-\frac{1}{4.21} x^{2}+\ldots
$$

que es la serie de Taylor de $f(x)=\sqrt{x+1}$ en un entorno de $x=0$.

Existen otros posibles desarrollos de la técnica principal. Podemos citar en primer lugar at utilizacion paro resolver ecuaciones lineales de segundo orden con coeficientes polinómicos (como por ejemplo las ectuaciones de Bessel) a ecuaciones integrales. Se pueden entonces poner muy fácilmente en evidencia las limitaciones de esta técnion con ecuaciones diferenciales no lineales de primer onden (del tipo $y^{\prime}=1+y^{2}, y y^{\prime}=1$, etc.).

Las dificultades ligadas a ta resolución de las relaciones de recurrencia obtenidas nos pereden sugenir uno importante variación de la técnica principal que ampliaró en una nuseva dirección el cumpo de problemas abordado. Se trata, en cierto modo, de invertir el proceso: en lugar de buscar las soluciones analítions de ecuaciones diferenciales mediante ia resolución de relaciones recurrentes, se trotaría de intentar resolver ciertas relaciones de recurrencia asociándolas a couraciones diferenciales de las que conocemos la solvción. Surge entonces una nuevs técnica, llamada técnica de la función generatriz.

Ejemplo: Dada una constante $\alpha \in R$, hallar el término general de la sucesion:

$$
\text { (*) }\left\{\begin{array}{l}
(n+1) a_{n+2}=(\alpha+n) a_{n} \\
a_{0}=1
\end{array}\right.
$$

Se multiplican por $x^{n}$ los dos miembros de la igualdad $(n+1) a_{n+1}=(\alpha+n) a_{n}$ y se suman desde 0 hasta $\infty$. Queda entonces:

$$
\sum_{n \geq 0}(n+1) a_{n+1} x^{n}=\alpha \sum_{n \geq 0} a_{n} x^{n}+x \sum_{n \geq 0} n a_{n} x^{n-1}
$$

0, lo que equivalente: $y^{\prime}(x)=\alpha y(x)+x y^{\prime}(x)$.

La ecuación diferencial asociada a la relación de recurrencia $(*)$ es:

$$
(1-x) y^{\prime}=\alpha y, \quad \text { con condición inicial } y(0)=1 \text {. }
$$

Supongamos que sabemos que la solución de esta ecuación es $y(x)=\frac{1}{(1-x)^{2}}$ y que conocemos el desarrollo en serie de Taylor de esta función:

$$
\frac{1}{(1-x)^{\alpha}}=\sum_{n \geq 0}\left(\begin{array}{c}
\alpha+n-1 \\
n
\end{array}\right) x^{n}
$$

Entonces el término general de la sucesión recurrente es:

$$
a_{n}=\left(\begin{array}{c}
\alpha+n-1 \\
n
\end{array}\right) \quad \forall n \geq 0 .
$$

